\title{
Combined high-resolution laser spectroscopy and nuclear decay spectroscopy for the study of the low-lying states in ${ }^{206} \mathrm{Fr},{ }^{202} \mathrm{At}$, and ${ }^{198} \mathrm{Bi}$
}

\author{
K. M. Lynch, ${ }^{1,2,{ }^{*}}$ T. E. Cocolios, ${ }^{2,3}$ J. Billowes, ${ }^{3}$ M. L. Bissell, ${ }^{2,3}$ I. Budinčević, ${ }^{2}$ T. Day Goodacre,,${ }^{3,4}$ R. P. de Groote, ${ }^{2}$ \\ G. J. Farooq-Smith, ${ }^{2,3}$ V. N. Fedosseev, ${ }^{4}$ K. T. Flanagan, ${ }^{3}$ S. Franchoo, ${ }^{5}$ R. F. Garcia Ruiz, ${ }^{2}$ H. Heylen, ${ }^{2}$ R. Li,${ }^{5}$ B. A. Marsh, ${ }^{4}$ \\ G. Neyens, ${ }^{2}$ R. E. Rossel ${ }^{4,6}$ S. Rothe, ${ }^{4}$ H. H. Stroke, ${ }^{7}$ K. D. A. Wendt, ${ }^{6}$ S. G. Wilkins,${ }^{3}$ and X. Yang ${ }^{2}$ \\ ${ }^{1}$ ISOLDE, PH Department, CERN, CH-1211 Geneva 23, Switzerland \\ ${ }^{2}$ KU Leuven, Instituut voor Kern- en Stralingsfysica, B-3001 Leuven, Belgium \\ ${ }^{3}$ School of Physics and Astronomy, The University of Manchester, Manchester M13 9PL, United Kingdom \\ ${ }^{4}$ EN Department, CERN, CH-1211 Geneva 23, Switzerland \\ ${ }^{5}$ Institut de Physique Nucléaire d'Orsay, F-91406 Orsay, France \\ ${ }^{6}$ Institut für Physik, Johannes Gutenberg-Universität Mainz, D-55128 Mainz, Germany \\ ${ }^{7}$ Department of Physics, New York University, New York, New York 10003, USA
}

(Received 25 November 2015; published 27 January 2016)

\begin{abstract}
High-resolution laser spectroscopy was performed on ${ }^{206} \mathrm{Fr}$ with the collinear resonance ionization spectroscopy (CRIS) experiment at CERN-ISOLDE. The hyperfine structure and isotope shift of the ground, first isomeric and second isomeric states were measured. The hyperfine components were unambiguously assigned to each nuclear state by means of laser-assisted nuclear decay spectroscopy. The branching ratios in the $\alpha$ decay of ${ }^{206} \mathrm{Fr}$ and ${ }^{202}$ At were also measured for the first time with isomerically purified beams. The extracted hindrance factors allow determination of the spin of the ground, first isomeric, and second isomeric states in ${ }^{202}$ At and ${ }^{198} \mathrm{Bi}$.
\end{abstract}

DOI: 10.1103/PhysRevC.93.014319

\section{INTRODUCTION}

Isotopes in the vicinity of the $Z=82$ magic shell closure display a wide variety of nuclear shapes and configurations. Close to the $N=126$ shell closure, spherical shapes are the most common. In the neutron-rich isotopes close to $N=136$, octupole-deformed nuclei are found [1,2]. In the neutrondeficient isotopes, the pairing force and the proton-neutron interaction work together to generate quasidegenerate nuclear configurations at low energy, often with very different spinparities, which results in the phenomenon of shape coexistence [3]. One striking example of shape coexistence occurs in ${ }^{186} \mathrm{~Pb}$, where the ground state is spherical, while the first two excited states are believed to be oblate and prolate [4,5]. In the ${ }_{80} \mathrm{Hg}$ isotopes a similar effect is observed, with the coexisting shapes approaching the ground state at $N=102[6,7]$. The variety of nuclear configurations in this region impacts the nuclear shape as indicated by the changes in the charge radii: the large odd-even staggering in the ${ }_{80} \mathrm{Hg}$ isotopes around $N=102$ [8] and the progressive departure from sphericity in the ${ }_{84} \mathrm{Po}$ isotopes for $N \leqslant 116[9,10]$. These effects occur in a region where isomers in odd- $N$ isotopes are frequent, arising from the near-degeneracy of the $v 3 p_{3 / 2}$ and $v 1 i_{13 / 2}$ orbitals [11].

Similar effects are observed in the odd- $Z$ isotopes, e.g., the $\pi 1 h_{9 / 2}$ intruder configuration around $N=104$ [12] and the

\footnotetext{
*kara.marie.lynch@cern.ch

Published by the American Physical Society under the terms of the Creative Commons Attribution 3.0 License. Further distribution of this work must maintain attribution to the author(s) and the published article's title, journal citation, and DOI.
}

large change in charge radii in the ${ }_{79} \mathrm{Au}$ isotopes at $N=107$ [13]. On the other side of the $Z=82$ shell closure, the $\pi 1 h_{9 / 2}$ configuration becomes the spherical-shell-model ground state and is in competition with an intruder $\pi 3 s_{1 / 2}$ configuration, corresponding to the excitation of a proton across the shell closure. This intruder configuration becomes the ground state in ${ }^{185} \mathrm{Bi}[14]$ and ${ }^{195} \mathrm{At} \mathrm{[15]} \mathrm{and} \mathrm{is} \mathrm{suggested} \mathrm{for}{ }^{197,199} \operatorname{Fr}[16,17]$.

The number of available nuclear configurations in this region of the nuclear chart translates into complex low-energy structures in the nuclei. In ${ }_{87}^{206} \mathrm{Fr}_{119}$, three states are competing at low energy. They have been identified from decay-spectroscopy studies [18], but the similarity in the nuclear properties of the two longest-lived states, both with half-lives of $15.9 \mathrm{~s}$ and $\alpha$-decay energies of $6792 \mathrm{keV}$, did not allow the unambiguous study of the different nuclear configurations. Laser spectroscopy studies have since confirmed the existence of the three states and provided additional information $[19,20]$, but have not yet been able to provide the full understanding of the nucleus. This isotope is also used in the high-precision study of the hyperfine anomaly by means of atom trapping [21,22].

In order to study the details of the structure of ${ }^{206} \mathrm{Fr}$, we have performed an experiment with the collinear resonance ionization spectroscopy (CRIS) experiment at the CERNISOLDE facility [23]. The CRIS technique allows the study of the hyperfine structure of short-lived isotopes produced online, whereby using ground-state or isomer selective ionization as a purification step, decay spectroscopy can be performed in clean conditions [19]. The recent development of highresolution CRIS with chopped cw laser light [24] offers a greater precision on the hyperfine parameters and isotope shift, and provides a greater selectivity of the isomers for decay-spectroscopy studies. By using these developments at 
the CRIS experiment, we have been able to study the $3^{(+)}, 7^{(+)}$, and $10^{(-)}$states in ${ }^{206} \mathrm{Fr}$.

In this article, we present the study of ${ }^{206} \mathrm{Fr}$ using highresolution laser spectroscopy and laser-assisted nuclear decay spectroscopy. The combination of the two methods allows the unambiguous assignment of hyperfine-structure peaks to each of the low-lying states of ${ }^{206}$ Fr. From this study, we have revealed the misidentification of the $10^{(-)}$state during the previous low-resolution laser spectroscopy measurements [19]. Here, we present the corrected hyperfine-structure values (and deduced electromagnetic moments) for the $10^{(-)}$state, alongside those for the $3^{(+)}$and $7^{(+)}$states, for the $7 s^{2} S_{1 / 2} \rightarrow$ $8 p^{2} P_{3 / 2}$ transition. In addition, we present the laser-assisted nuclear decay spectroscopy study of the ground state and isomers in ${ }^{206} \mathrm{Fr},{ }^{202} \mathrm{At}$, and ${ }^{198} \mathrm{Bi}$.

\section{EXPERIMENTAL TECHNIQUE}

The francium isotopes were produced at the CERN ISOLDE facility [25] by $1.4 \mathrm{GeV}$ proton-induced spallation on a thick uranium-carbide target. The atoms were ionized on the hot $(2400 \mathrm{~K})$ surface of a tantalum capillary tube and extracted from the target-ion source assembly at $40 \mathrm{keV}$. The ${ }^{206} \mathrm{Fr}$ isotopes of interest were mass selected with the high-resolution mass separator (HRS) and injected into the radio-frequency ISOLDE cooler and buncher, ISCOOL. The bunched ion-beam was extracted at $100 \mathrm{~Hz}$ and deflected to the CRIS experimental beam line [26-28]. Collisional neutralization with potassium vapor in a hot charge-exchange cell $(500 \mathrm{~K})$ was used, and the non-neutralized component of the beam was deflected away. The atomic bunch was passed through a differential-pumping section and overlapped in time and space with two copropagating laser pulses in the 1.2-m-long interaction region. The region was held at less than $10^{-9}$ mbar to minimize the background produced from nonresonant collisional ionization.

The francium atoms were resonantly excited via the $7 s^{2} S_{1 / 2} \rightarrow 8 p^{2} P_{3 / 2}$ transition (23658.306 $\mathrm{cm}^{-1}$ [29]) with use of $422.7 \mathrm{~nm}$ laser light. The $100 \mathrm{~mW} \mathrm{cw} \mathrm{laser} \mathrm{light}$ was produced by frequency doubling the fundamental laser light $(2.7 \mathrm{~W})$ from a Matisse TS continuous wave Ti:Sa laser (pumped by a Coherent Verdi G18 $15 \mathrm{~W}$ laser) using a Wavetrain external cavity frequency doubler. The frequency of the fundamental laser light from the Matisse laser was continuously measured with a HighFinesse WSU2 wavelength meter, calibrated with reference to a temperature-stabilized $\mathrm{HeNe}$ laser. Short pulses of light were produced from the continuous-wave laser by switching the laser-light polarization using a Pockels cell and passing it through a polarizing beam splitter cube. Fast switching of the high voltage $2.4 \mathrm{kV}$ (inducing a $\pi / 2$ rotation) applied to the Pockels cell was possible with use of a Behlke FSWP91-01 fast square-wave pulser. Half-wave plates and polarizers before and after the Pockels cell further enhanced the on-off ratio of the 100-ns laser pulse to $1: 180$. The frequency of the $422.7 \mathrm{~nm}$ laser light was scanned using the Matisse Commander software, in order to probe the hyperfine splitting of the $7 s^{2} S_{1 / 2} \rightarrow 8 p^{2} P_{3 / 2}$ atomic transition.
The nonresonant ionization step from $8 p^{2} P_{3 / 2}$ across the ionization threshold was delivered by 1064-nm laser light (13 W) produced from a Litron LPY 601 50-100 PIV Nd:YAG laser at a repetition rate of $100 \mathrm{~Hz}$. Arrival of the $422.7 \mathrm{~nm}$ and $1064 \mathrm{~nm}$ laser-light pulses in the interaction region was controlled using a Quantum Composers QC9258 digital delay pulse generator. The optimum balance between linewidth and laser-ionization efficiency was obtained when the pulse length of the $422.7 \mathrm{~nm}$ resonant excitation step was $100 \mathrm{~ns}$ and the $1064 \mathrm{~nm}$ ionization step was delayed by $100 \mathrm{~ns}$ with respect to the start of the resonant pulse [24]. The power of the $422.7 \mathrm{~nm}$ and $1064 \mathrm{~nm} \mathrm{cw}$ laser light entering the beamline was measured to be $25 \mathrm{~mW}$ and $8 \mathrm{~W}$, respectively, enough to saturate both transitions. The two laser pulses were overlapped spatially with the atomic bunch and induced stepwise excitation and ionization. The resulting resonant ions impinged on a copper plate and the corresponding secondary electrons were detected with an off-axis multichannel plate (MCP). The signals from the MCP were amplified, discriminated, and read by a National Instruments USB-6211 DAQ card. A computer running a Python-based data acquisition and control program (triggered by the Quantum Composers pulse generator at a rate of $10 \mathrm{~Hz}$ ) recorded the number of ions counted with the DAQ card, alongside the frequency of the $422.7 \mathrm{~nm}$ resonant-excitation step [28].

An alternative detection method was employed when identification of the resonant ions was necessary. By changing several electrostatic elements in the CRIS beam line, the resonant ions could be deflected instead to the Decay Spectroscopy Station (DSS), which housed a rotatable-wheel implantation system [30]. The resonant ions passed through an annular Canberra passivated implanted planar silicon (APIPS) detector (BKANPD 300-18 RM, thickness $300 \mu \mathrm{m}$ ) with an aperture of $4 \mathrm{~mm}$. A collimator placed before the APIPS detector protects the detector from direct implantation. The resonant ions were implanted into one of eight carbon foils [20(1) $\left.\mu \mathrm{g} \mathrm{cm}^{-2}\right]$ and their $\alpha$-particle emissions detected by the APIPS detector in front or by another PIPS detector (BKA 300-17 AM, thickness $300 \mu \mathrm{m})$ situated behind. The two detectors are placed at specific distances away from the carbon foils, in order to obtain the optimum solid angle coverage of $65 \%$, estimated with GEANT4 calculations [31]. The PIPS detectors were connected to Canberra 2003BT preamplifiers via custom-made microdot cables to SMA electrical feedthroughs. The steel wheel could be rotated and placed in ten different positions between the PIPS detectors. Positions 1 to 8 allowed for implantation of the ions into the carbon foils. The ninth position holds a Faraday cup that acted as a beam monitoring device to tune (stable) beam to the implantation site. The tenth position held two $50 \mathrm{~Bq}$ open ${ }^{241} \mathrm{Am} \alpha$-decay sources (one pointing in the direction of the APIPS detector, the other towards the PIPS detector). The $\alpha$-decay sources allowed offline testing, optimization, and calibration of the PIPS detectors and acquisition system. The signals from the PIPS detectors were acquired with XIA digital gamma finder (DGF) modules, revision D. One module was dedicated to signals from the PIPS detectors, with a sampling rate of $100 \mathrm{~ns}$. The second module received trigger signals to correlate the ion-bunch release from ISCOOL and the firing of the two laser-light 

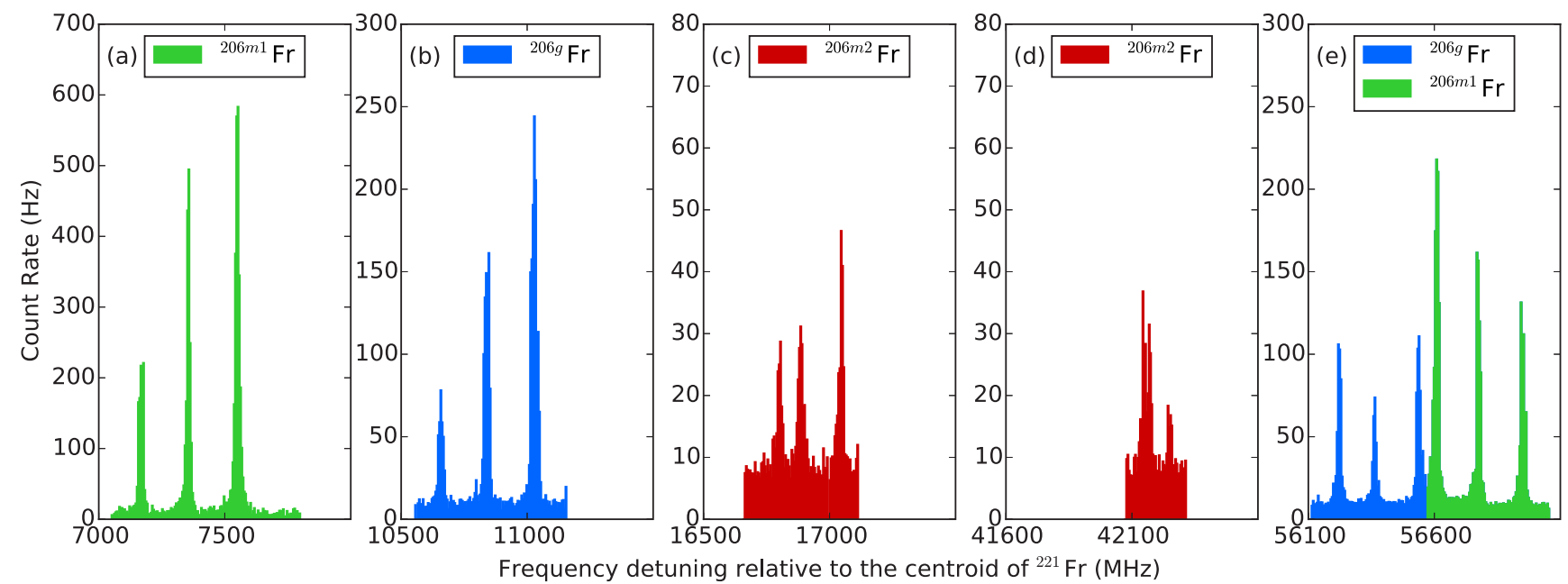

FIG. 1. Collinear resonance ionization spectroscopy of the low lying states of ${ }^{206} \mathrm{Fr}$, relative to the centroid frequency of ${ }^{221} \mathrm{Fr}$. The spin $3^{(+)}$state is shown in blue [(b) and (e)], the spin $7^{(+)}$state is shown in green [(a) and (e)], and the spin $10^{(-)}$state is in red [(c) and (d)].

pulses. Each DGF channel was optimized for the incoming signal by modifying the on-board parameters. Pulse height analysis was made on-line and the resulting pulse-height and timing information were recorded.

\section{RESULTS}

\section{A. Decay-assisted laser spectroscopy}

The hyperfine-structure spectra of the low-lying states in ${ }^{206} \mathrm{Fr}$ for the resonant $7 s^{2} S_{1 / 2} \rightarrow 8 p^{2} P_{3 / 2}$ transition are shown in Fig. 1. The laser frequency is plotted relative to the centroid frequency of the reference isotope, ${ }^{221} \mathrm{Fr}$. Six groups of three hyperfine components are identified, associated in pairs to each state in ${ }^{206} \mathrm{Fr}$ : Figs. 1(b) and 1(e) show the hyperfine structure of ${ }^{206 g}$ Fr, Figs. 1(a) and 1(e) show the structure of ${ }^{206{ }^{m} 1} \mathrm{Fr}$, and Figs. 1(c) and 1(d) show that of ${ }^{206 m 2} \mathrm{Fr}$. The linewidth of the ${ }^{206} \mathrm{Fr}$ spectra was determined to be $\sim 20$ (1) $\mathrm{MHz}$, with a background of $<10 \mathrm{~Hz}$. The groups were identified by locking the frequency of the laser to a hyperfine-structure resonance and deflecting the resonantly ionized beam to the DSS. From the $\alpha$-particle decay energies characteristic of the $3^{(+)}, 7^{(+)}$, and $10^{(-)}$states in ${ }^{206} \mathrm{Fr}$, each resonance peak could be identified as one of the three states.

The identification of the hyperfine structures is hindered by the degeneracies in the $\alpha$-decay pattern of ${ }^{206} \mathrm{Fr}$ (see Fig. 2), as both the $3^{(+)}$and $7^{(+)}$states have similar half-lives $(15.9 \mathrm{~s})$, $\alpha$-decay energies (6792 keV), and branching ratios (84\%) [18]. This situation is similar to ${ }^{202} \mathrm{Fr}$, where the $\alpha$-decay pattern of its daughter nucleus ${ }^{198}$ At had to be relied upon [19]. The $\alpha$ decay energy spectra for the outer $\left({ }^{206 m 1} \mathrm{Fr}\right)$, middle $\left({ }^{206 g} \mathrm{Fr}\right)$, and inner $\left({ }^{206 m 2} \mathrm{Fr}\right)$ hyperfine-structure components of ${ }^{206} \mathrm{Fr}$ are shown in Fig. 3. The identified peaks arise from ${ }^{206} \mathrm{Fr}$ and ${ }^{206} \mathrm{Rn}$ via the $\beta$ decay of ${ }^{206} \mathrm{Fr}$, and from ${ }^{202}$ At via the $\alpha$ decay of ${ }^{206} \mathrm{Fr}$.

The red spectrum of Fig. 3 is related to the innermost hyperfine components [see Figs. 1(c) and 1(d)] and shows an excess of $\alpha$ particles at $6930 \mathrm{keV}$, characteristic of the decay of the $10^{(-)}$state in ${ }^{206} \mathrm{Fr}$. This state also decays to the $7^{(+)}$ state, and therefore the spectrum displays common features with the other $\alpha$-decay energy spectra. The blue spectrum related to the intermediate hyperfine components [Figs. 1(b) and 1(e)] shows an excess of $\alpha$ particles at $6228 \mathrm{keV}$, while the green spectrum related to the outermost components [Figs. 1(a) and 1(e)] shows an excess of $\alpha$ particles at $6135 \mathrm{keV}$. This arises from the $\alpha$ decay of the $\left(2,3^{+}\right)$and the $\left(7^{+}\right)$states in ${ }^{202} \mathrm{At}$, respectively. Those are produced directly from the $\alpha$ decay of the $3^{(+)}$and $7^{(+)}$states in ${ }^{206}$ Fr respectively, hereby establishing the assignment of each hyperfine structure group to the states in ${ }^{206} \mathrm{Fr}$. This analysis is additionally carried over each hyperfine peak separately and confirms the grouping

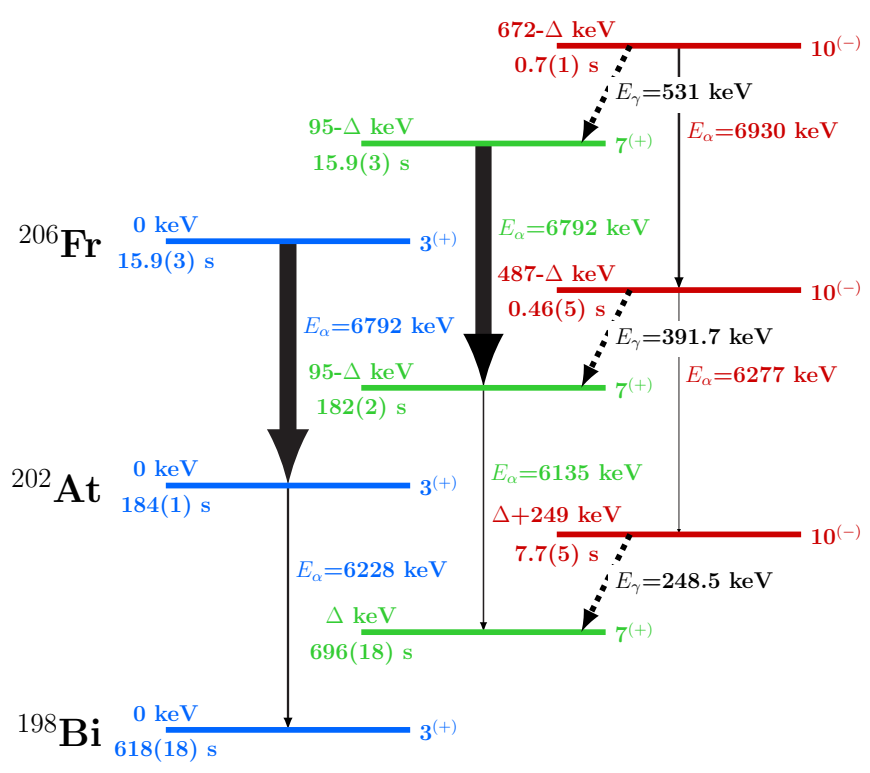

FIG. 2. $\alpha$-decay pattern of the ${ }^{206} \mathrm{Fr}-{ }^{202} \mathrm{At}-{ }^{198} \mathrm{Bi}$ decay chain [18]. Only the transitions relevant to the $\alpha$-decay tagging of the hyperfine components are presented. The dashed arrows represent $\gamma$ decay and the full arrows $\alpha$ decay, whereby the width is proportional to the branching ratio. Note the spins of ${ }^{202 g} \mathrm{At}$ and ${ }^{198 g} \mathrm{Bi}$ are the newly assigned $3^{(+)}$, instead of $\left(2,3^{+}\right)$. 


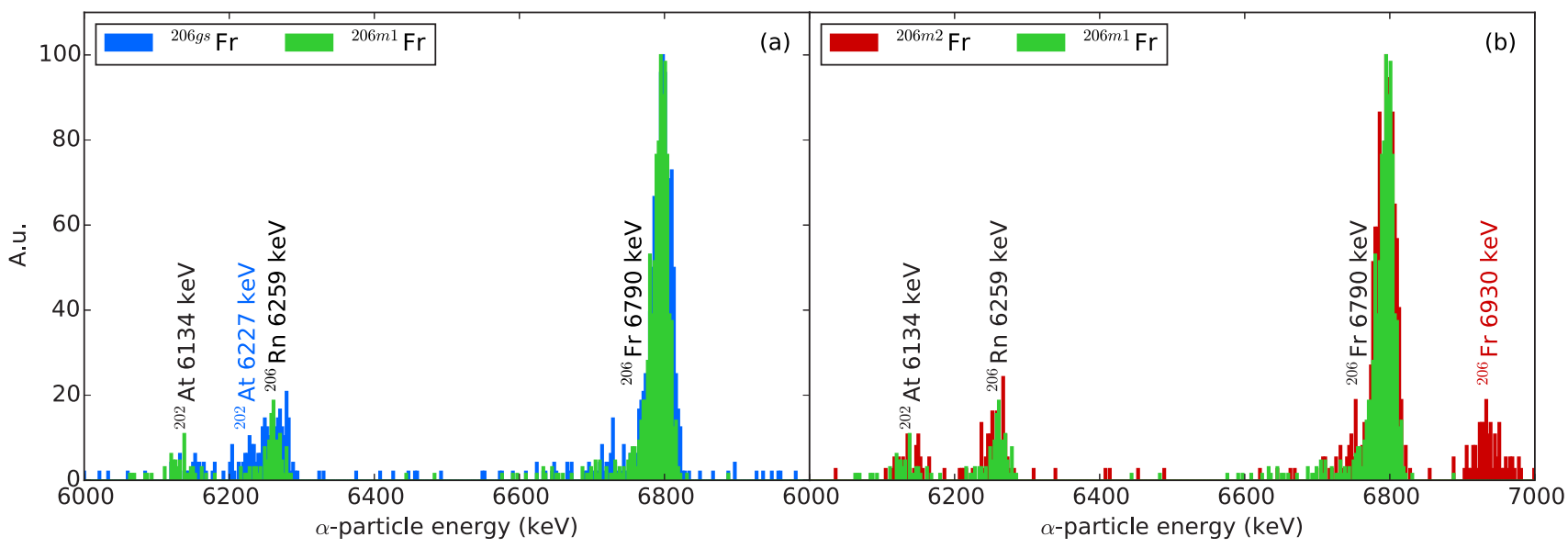

FIG. 3. $\alpha$-decay energy spectra of laser-separated beams of ${ }^{206} \mathrm{Fr}$ and its isomers, normalised to the $6790 \mathrm{keV}$ peak. (a) Hyperfine components of laser-separated ${ }^{206 g} \mathrm{Fr}$ and ${ }^{206 m 1} \mathrm{Fr}$. The excess in $\alpha$ particles at $6135 \mathrm{keV}(6228 \mathrm{keV}$, respectively), is characteristic of the decay of the $\left(7^{+}\right)\left[\left(2,3^{+}\right)\right.$, respectively] state in ${ }^{202}$ At, arising from the decay of the $7^{(+)}\left(3^{(+)}\right.$, respectively) state in ${ }^{206} \mathrm{Fr}$. (b) Hyperfine components of ${ }^{206 m 1} \mathrm{Fr}$ and ${ }^{206 m 2} \mathrm{Fr}$. The excess in $\alpha$ particles at $6930 \mathrm{keV}$ is characteristic of the decay of the $10^{(-)}$state in ${ }^{206} \mathrm{Fr}$.

of the hyperfine components based on angular-momentum coupling considerations.

Table I presents the hyperfine structure $A$ and $B$ factors, isotope shift, magnetic dipole moment, electric quadrupole moment, and change in mean-square charge radii of ${ }^{206} \mathrm{Fr}$ for the $7 s^{2} S_{1 / 2} \rightarrow 8 p^{2} P_{3 / 2}$ transition, relative to ${ }^{221} \mathrm{Fr}$. The magnetic dipole moments were determined via the known ratio

$$
\mu=\frac{I A}{I_{\mathrm{ref}} A_{\mathrm{ref}}} \mu_{\mathrm{ref}},
$$

with reference to the magnetic moment measurement of ${ }^{210} \mathrm{Fr}$ [33] for the $A\left(7 s^{2} S_{1 / 2}\right)$ factor only [34], due to its lower relative uncertainty. The quoted uncertainty on the magnetic moments is dominated by the uncertainty on the ${ }^{210} \mathrm{Fr}$ magnetic moment [33]. This magnetic moment of $\mu\left({ }^{210} \mathrm{Fr}=\right.$ 4.38(5)) $\mu_{N}$ is deduced from the measured hyperfine splitting of the $9 s^{2} S_{1 / 2}$ level and a calculated hyperfine field, and presents the most accurate ground-state moment measurement to date in this isotopic chain.

The quadrupole moments for the nuclear states were calculated from a theoretical evaluation of the electric field gradient, since no absolute measurement of the electric quadrupole moment is available. Many-body calculations using the coupled-cluster $\left(\mathrm{CCSD}_{\mathrm{t} 3}\right)$ method [35] give

$$
\frac{B}{Q_{s}}=84.01 \mathrm{MHz} / \mathrm{b}
$$

for the $8 p^{2} P_{3 / 2}$ atomic state. Since no error is available for $B / Q_{s}$, the uncertainty on the quadrupole moments comes directly from the statistical uncertainty associated with our $B\left(8 p^{2} P_{3 / 2}\right)$ factor.

The changes in the mean-square charge radii were extracted using the most recent atomic calculations [36] and a King plot

TABLE I. Nuclear spin, hyperfine structure parameters of the $7 s^{2} S_{1 / 2}$ and $8 p^{2} P_{3 / 2}$ atomic levels, isotope shift of the $422.7 \mathrm{~nm}$ transition, electromagnetic moments of ${ }^{206} \mathrm{Fr}$, and changes in the mean square charge radii from this work, our previous work [19], and that of Voss et al. $[20,32]$. The magnetic moment values were extracted from the $A\left(7 s^{2} S_{1 / 2}\right)$ state only. For the charge radii, the statistical uncertainties are followed by the systematic uncertainties, propagated from ${ }^{221} \mathrm{Fr}$ for all data sets. See the Discussion section for a detailed consideration of the

\begin{tabular}{|c|c|c|c|c|c|c|c|c|c|}
\hline Isotope & Spin & $\begin{array}{c}A\left(7 s^{2} S_{1 / 2}\right) \\
(\mathrm{MHz})\end{array}$ & $\begin{array}{c}A\left(8 p^{2} P_{3 / 2}\right) \\
(\mathrm{MHz})\end{array}$ & $\begin{array}{c}B\left(8 p^{2} P_{3 / 2}\right) \\
(\mathrm{MHz})\end{array}$ & $\begin{array}{c}\delta v^{221-206} \\
(\mathrm{MHz})\end{array}$ & $\begin{array}{c}\mu \\
\left(\mu_{N}\right)\end{array}$ & $\begin{array}{l}Q_{S} \\
\text { (b) }\end{array}$ & $\begin{array}{c}\delta\left\langle r^{2}\right\rangle^{221-206} \\
\quad\left(\mathrm{fm}^{2}\right)\end{array}$ & Ref. \\
\hline \multirow[t]{3}{*}{${ }^{206 g} \mathrm{Fr}$} & $3^{(+)}$ & $13057.8(10)$ & $47.5(10)$ & $-29.8(10)$ & $30445.6(14)$ & $+3.97(5)$ & $-0.354(8)$ & $-1.4851(1)(162)$ & this work \\
\hline & & 13120(30) & & & $30040(120)$ & $+3.99(5)$ & & $-1.465(6)(16)$ & [19] \\
\hline & & $13052.2(18)$ & & & & $+3.91(3)$ & $-0.253(18)$ & $-1.4748(1)(147)^{\mathrm{a}}$ & [20] \\
\hline \multirow[t]{2}{*}{${ }^{206 m 1} \mathrm{Fr}$} & $7^{(+)}$ & $6613.2(10)$ & $24.0(10)$ & $-12.1(10)$ & $30485.0(10)$ & $+4.70(5)$ & $-0.143(11)$ & $-1.4870(1)(162)$ & this work \\
\hline & & $6616.0(7)$ & & & & $+4.68(4)$ & $-0.138(17)$ & $-1.4772(1)(148)^{\mathrm{a}}$ & [20] \\
\hline \multirow[t]{2}{*}{${ }^{206 m 2} \mathrm{Fr}$} & $10^{(-)}$ & $2416.0(10)$ & $8.8(10)$ & $105.5(24)$ & $29005.4(14)$ & $+2.45(3)$ & $+1.255(28)$ & $-1.4154(1)(155)$ & this work \\
\hline & & $2416.1(4)$ & & & & $+2.44(2)$ & $+1.307(9)$ & $-1.4048(1)(140)^{\mathrm{a}}$ & [20] \\
\hline
\end{tabular}
spin assignments.

${ }^{\mathrm{a} B a s e d}$ on the quoted systematic uncertainty of $1 \%$. 
TABLE II. Decay properties of the ground and long-lived isomeric states in ${ }^{206} \mathrm{Fr}$ and ${ }^{202}$ At. The ${ }^{206} \mathrm{Fr}$ nuclear spins have been measured by Voss et al. [20] and confirmed in this work; the ${ }^{202}$ At nuclear spins are then determined from $\alpha$ decay in this work. The half-lives, $\alpha$-particle energies, and relative intensities are extracted from the work of Huyse et al. [18]. The branching ratios are from this work, with those of Huyse et al. [18] shown for comparison. The partial decay widths are extracted based on the data in this table and the hindrance factors estimated based on the partial decay widths of ${ }^{204,206} \mathrm{Rn}$ for ${ }^{206} \mathrm{Fr}$, and those of ${ }^{200,202} \mathrm{Po}$ for ${ }^{202} \mathrm{At}$.

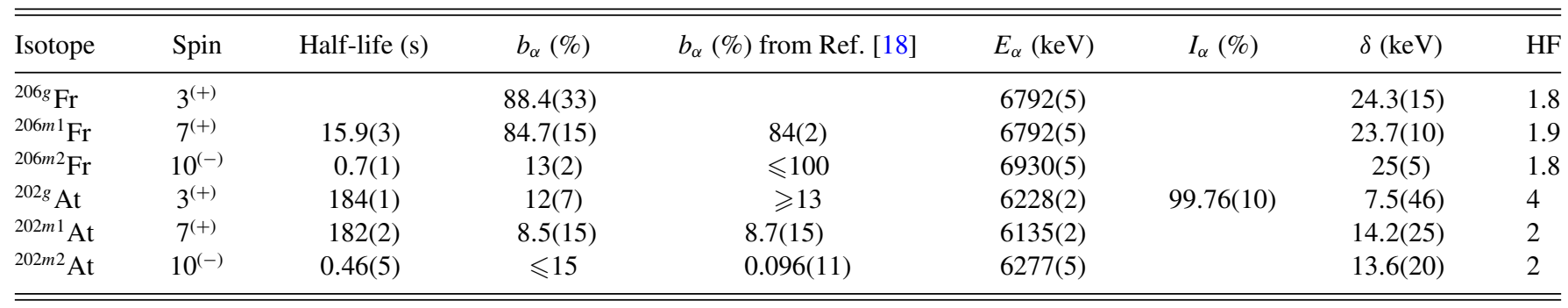

analysis [19], using the equation

$$
\delta v=\frac{A-A_{\mathrm{ref}}}{A \times A_{\mathrm{ref}}} M_{422}+F_{422} \delta\left\langle r^{2}\right\rangle^{A A_{\mathrm{ref}}},
$$

with $M_{422}=750(330) \mathrm{GHz} / \mathrm{amu}$ and $F_{422}=-20.67(21)$ $\mathrm{GHz} / \mathrm{fm}^{2}$ [19].

The results reported in Table I are within $2 \sigma$ agreement with the previous CRIS results [19] but are much more precise, thanks to the higher resolution of this work. Only the values for the $10^{(-)}$state are very different. This discrepancy is due to a systematic dip in the power of the scanning laser during the 2012 experiment over the frequency range where the left-hand multiplet of the $10^{(-)}$state was located. This resulted in the nonobservation of one of the peaks and therefore a wrong value assignment of the hyperfine components.

The results are also compared to the results from collinear laser spectroscopy by Voss et al. [20,32]. Their work uses a different transition at $718.0 \mathrm{~nm}\left(7 s^{2} S_{1 / 2} \rightarrow 7 p^{2} P_{3 / 2}\right)$, meaning only the atomic ground state hyperfine parameter $A\left(7 s^{2} S_{1 / 2}\right)$ can be directly compared, as shown in Table I. While the hyperfine parameters are in general agreement, the small differences are not negligible when compared to the high precision quoted for each value, representing a $2 \sigma$ difference for ${ }^{206 g} \mathrm{Fr}$ and ${ }^{206 m 1} \mathrm{Fr}$.

\section{B. Laser-assisted nuclear decay spectroscopy}

The $\alpha$-decay energy spectra are analyzed and the branching ratios in the decay of ${ }^{206}$ Fr extracted. The $\alpha$-to- $\beta / \gamma$ branching ratios of each long-lived state in ${ }^{206} \mathrm{Fr}$ are determined by comparing the number of $\alpha$-decay events at the energy characteristic of the state of interest to that of the $\alpha$-decay energy of the daughter nucleus by the competing process.

In the study of the $3^{(+)}$and $7^{(+)}$states in ${ }^{206} \mathrm{Fr}$, the $\alpha$-decay events at $6792 \mathrm{keV}$ are compared to those resulting from the $\alpha$ decay of ${ }^{206} \mathrm{Rn}$ at $6259 \mathrm{keV}$ [37], whereby ${ }^{206} \mathrm{Rn}$ is populated from the $\beta$ decay of ${ }^{206} \mathrm{Fr}$. Using beams of ${ }^{206} \mathrm{Fr}$ separated by CRIS, the beam purity is guaranteed by putting the first laser on resonance with one particular hyperfine transition, thus a single state is delivered to the DSS. The $\beta$ decay to ${ }^{206} \mathrm{Rn}$ thus only occurs from the state of interest, and all $\alpha$-decay events from ${ }^{206} \mathrm{Rn}$ originate from the $\beta$ decay of ${ }^{206} \mathrm{Fr}$. The number of $\beta$-decay events is corrected for the branching ratio in the $\alpha$ decay of ${ }^{206} \mathrm{Rn}, b_{\alpha}=62 \%$, and for the unobserved decays, as the observation period $\left(25 \mathrm{~min}\right.$ for $3^{(+)}$and 54 $\min$ for $7^{(+)}$) are of similar magnitude to the half-life of ${ }^{206} \mathrm{Rn}$ ( $T_{1 / 2}=5.67 \mathrm{~min}$ [38]). The recoils from $\beta$ decay are negligible and the detection efficiency for all $\alpha$-decay events is the same. The obtained branching ratios are $88.4(33) \%$ and $84.7(15) \%$, respectively, and are given in Table II. This represents the first branching-ratio measurement for the $\alpha$ decay of the $3^{(+)}$state. The branching ratio for the $7^{(+)}$state is in good agreement with the literature value of $84(2) \%$ [18].

In the case of the $10^{(-)}$state, the $\alpha$-decay events at $6930 \mathrm{keV}$ are compared to events arising from the internal transition to the $7^{(+)}$state, observed in the $\alpha$-decay events at $6792 \mathrm{keV}$. Those events are corrected for the newly determined branching ratio in the decay of the $7^{(+)}$state. No other corrections are necessary. A branching ratio of $13(2) \%$ is found. This is the first measurement of the branching ratio for the $10^{(-)}$decay.

The $\alpha$-decay energy spectra can also be used to determine the branching ratios in the decay of ${ }^{202}$ At. As only ${ }^{206} \mathrm{Fr}$ is implanted into the carbon foil, all the ${ }^{202}$ At events originate from the $\alpha$ decay of ${ }^{206} \mathrm{Fr}$. The number of ${ }^{202}$ At atoms can therefore be determined directly from the number of $\alpha$ particles observed. The recoil of the ${ }^{202}$ At nucleus after the $\alpha$ decay of ${ }^{206} \mathrm{Fr}$ can be sufficient for the daughter nucleus to exit the foil. This process has been highlighted in decay studies with similar detection setup geometries $[39,40]$. The decay of ${ }^{206} \mathrm{Fr}$ has been simulated using the SRIM simulation package for charged particles through matter [41], and a detailed analysis will be presented in a forthcoming paper [31]. $29 \%$ of the ${ }^{202}$ At recoils escape the carbon foil, and a third of these will be collected at the surface of the detector, with a $50 \%$ probability of detection of the subsequent $\alpha$ decay. Altogether, the normalized detection efficiency of decays from ${ }^{202} \mathrm{At}$ is then $51 \%$, instead of the nominal $65 \%$ for ${ }^{206} \mathrm{Fr}$.

Using the $\alpha$-decay energy spectra shown in Fig. 3, the branching ratios in the decay of the $\left(2,3^{+}\right),\left(7^{+}\right)$, and $\left(10^{-}\right)$ states in ${ }^{202} \mathrm{At}$ are determined by comparing the number of decaying events at energies $E_{\alpha}\left({ }^{202} \mathrm{At}\right)=6228,6135,6277 \mathrm{keV}$ and $E_{\alpha}\left({ }^{206} \mathrm{Fr}\right)=6792,6792,6930 \mathrm{keV}$, respectively (see Table II). The branching ratios are found to be $12(7) \%$ for the $\left(2,3^{+}\right)$ state and $8.5(15) \%$ for the $\left(7^{+}\right)$state, compared to $\geqslant 13 \%$ and $8.5(15) \%$ according to Huyse et al. [18]. The proximity of the ${ }^{206} \mathrm{Rn} \alpha$-decay peak at $6259 \mathrm{keV}$ and the high branching ratio towards this isotope in the decay of the $10^{(-)}$state in ${ }^{206} \mathrm{Fr}$ results in the difficult identification of the $6277 \mathrm{keV}$ peak in the 
decay of the $\left(10^{-}\right)$state in ${ }^{202} \mathrm{At}$; only an upper limit of $\leqslant 15 \%$ could be determined, compared to $0.096(11) \%$ from literature [18]. These results show a good agreement on the branching ratio of the $\left(7^{+}\right)$state and offer the first measurement of the branching ratio of the $\left(2,3^{+}\right)$state.

\section{DISCUSSION}

\section{A. Spins and moments of ${ }^{206} \mathrm{Fr}$}

The nuclear spin of the second isomeric state in ${ }^{206} \mathrm{Fr}$ has been a topic of recent discussion [20], leading to a determined spin of $10^{(-)}$. To aid this discussion using the $7 s^{2} S_{1 / 2} \rightarrow 8 p^{2} P_{3 / 2}$ transition probed in this experiment, the ratio of $A\left(7 S_{1 / 2}\right)$ to $A\left(8 P_{3 / 2}\right)$ can be calculated. This ratio is expected to be constant for all isotopes and isomers, with variations arising from hyperfine-anomaly effects of $\sim 1 \%$, which is below our uncertainty limit.

In Fig. 4(a), the calculated ratios obtained by assuming different nuclear spins when fitting the ${ }^{206 m 2}$ Fr hyperfine structure are presented. The suggested single-particle configuration of $\left(\pi 1 h_{9 / 2} \otimes v 1 i_{13 / 2}\right)$ means that nuclear spins of $I=2^{-}$to $11^{-}$are possible. The blue line represents the ratio of $A\left(7 S_{1 / 2}\right)$ to $A\left(8 P_{3 / 2}\right)$ for the ${ }^{206 g}$ Fr state, and similarly the green line presents the ratio for ${ }^{206 m 1}$ Fr. Figure 4(b) presents the resulting $g$ factors for different nuclear spins (again $I=2^{-}$to $11^{-}$) for ${ }^{206 m 2} \mathrm{Fr}$, alongside the established $g$ factors for the $\left(10^{-}\right)$state in ${ }^{202,204} \mathrm{Fr}$ (both located behind the

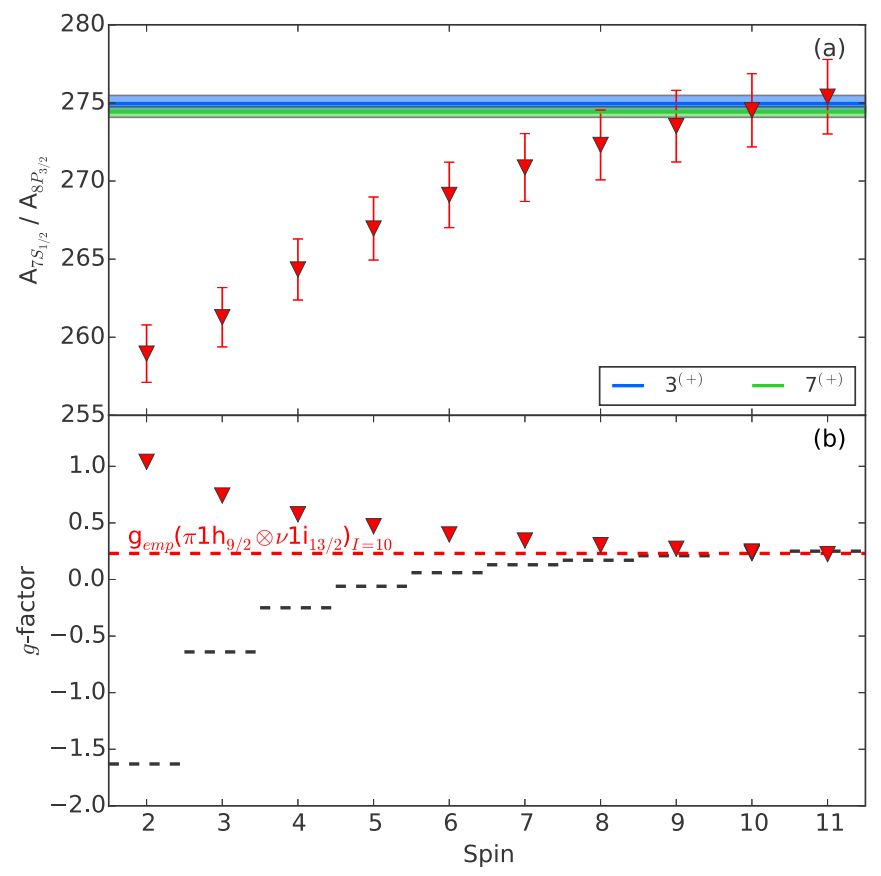

FIG. 4. (a) Ratio of the hyperfine $A$ factors for the $7 s^{2} S_{1 / 2}$ and $8 p^{2} P_{3 / 2}$ electronic states of ${ }^{206 m 2} \mathrm{Fr}$, for different nuclear spins $(I=$ $2^{-}$to $\left.11^{-}\right)$. The blue line represents the ratio for ${ }^{206 g} \mathrm{Fr}$ and the green line for ${ }^{206 m 1} \mathrm{Fr}$. (b) The expected $g$ factors for the different nuclear spins for ${ }^{206 m 2} \mathrm{Fr}$, alongside the established $g$ factors for the $\left(10^{-}\right)$ state in ${ }^{202,204} \mathrm{Fr}$. The dashed lines give the empirically calculated $g$ factor for each spin. See text for details. $\left(10^{-}\right)$data point for $\left.{ }^{206 m 2} \mathrm{Fr}\right)$. The uncertainties on all $g$ factors are smaller than the data points. The dashed lines illustrate the empirical $g$ factors for the different spins, calculated from the magnetic moments of the neighboring ${ }^{201 m}$ Po [42] and ${ }^{203} \mathrm{Fr}$ isotopes, in the same manner as our previous paper [19]. While the spread of the experimental and empirical $g$ factors is similar, the values progress in opposite directions as the spin goes from $11^{-}$to $2^{-}$. As Fig. 4 shows, spin $\left(9^{-}\right),\left(10^{-}\right)$, and $\left(11^{-}\right)$provide the closest agreement to $A$-ratio and $g$-factor values, but given the large uncertainty on these values; the determination based solely on our hyperfine-structure analysis cannot be considered definitive.

Previous decay studies have, however, identified the internal transition of the second isomer to the $7^{(+)}$state via an E3 transition [18]. As a consequence, only a spin of $4^{(-)}$or $10^{(-)}$is possible for this state, hereby reducing the number of possibilities. In light of the previous observations on Fig. 4 and considering that a $4^{(-)}$state would not be isomeric against the $3^{(+)}$ground state, it can be unambiguously concluded that the spin of the second isomer in ${ }^{206} \mathrm{Fr}$ is $10^{(-)}$.

The magnetic dipole moments from this work and from Voss et al. [20,32], shown in Table I, are in good agreement, despite the difference in the hyperfine parameter for the atomic ground state. This is because the uncertainty on the magnetic dipole moment is dominated by the magnetic moment of the reference isotopes, rendering the difference in ${ }^{206}$ Fr hyperfine parameters negligible. Similarly, the results from the high-resolution study agree with the results from the low-resolution study [19], and all the conclusions presented previously are still valid, namely that the experimental $g$ factors are consistent with the empirical $g$ factors, showing that the nuclear configurations of the long-lived states in ${ }^{206} \mathrm{Fr}$ are rather pure, corresponding to $\left(\pi 1 h_{9 / 2} \otimes \nu 3 p_{3 / 2}\right)_{3^{+}}$, $\left(\pi 1 h_{9 / 2} \otimes v 2 f_{5 / 2}\right)_{7^{+}}$, and $\left(\pi 1 h_{9 / 2} \otimes v 1 i_{13 / 2}\right)_{10^{-}}$. Note that the corrected value for ${ }^{206 m 2} \mathrm{Fr}$ is also in good agreement with the empirical $g$ factor [19].

The electric quadrupole moments are generally in good agreement, coinciding well with the neighboring nuclei for ${ }^{206 g} \mathrm{Fr}$ and ${ }^{206 m 1} \mathrm{Fr}$. The quadrupole moments of ${ }^{206 g, m 1} \mathrm{Fr}$ agree well with Refs. [20,32], respectively. ${ }^{206 m 2} \mathrm{Fr}$ has a large quadrupole moment, as expected from a rigidly deformed nucleus, and is in broad agreement $(1.4 \sigma)$ with Ref. [20].

The difference in the hyperfine parameters may, however, matter in studies where high-precision data are required, such as the work on the hyperfine anomaly by Zhang et al. [22]. In that work, the hyperfine splitting of the $7 P_{1 / 2}$ atomic state is measured down to $10 \mathrm{ppm}$ and compared to the hyperfine splitting in the $7 S_{1 / 2}$ atomic state. A difference of $5000 \mathrm{ppm}$ between our work and that of Voss et al. (see Table I) may therefore have an impact on the analysis of the hyperfine anomaly.

Finally, the changes in the mean-square charge radii are in good agreement within the large systematic uncertainties arising from the $F_{422}$ and $M_{422}$ atomic parameters [19,36]. As seen from Eq. (3), the systematic uncertainty scales almost linearly with the mass difference and isotope shift. A more stringent comparison of the available data can therefore be extracted from the changes in the mean-square charge radii between the ground and isomeric states, as the mass effects vanish and the isotope shifts are typically smaller. 
The isomer shift for ${ }^{206 m 1} \mathrm{Fr}$ with respect to ${ }^{206 g} \mathrm{Fr}$ then gives $-0.0019(1)(1) \mathrm{fm}^{2}$ from this work and $-0.0024(1)(1) \mathrm{fm}^{2}$ for Voss et al. The isomer shift for ${ }^{206 m 2} \mathrm{Fr}$ gives $0.0697(1)(7) \mathrm{fm}^{2}$ from this work and $0.0700(1)(7) \mathrm{fm}^{2}$ for Voss et al. These values are in good agreement and show that the discrepancies arise from the systematic effects induced by the atomic parameters.

When comparing the changes in the mean-square charge radii to neighboring isotopes, it was concluded in our previous work [19] that ${ }^{206 g} \mathrm{Fr}$ and ${ }^{206 m 1} \mathrm{Fr}$ follow the same trend as the isotonic lead isotopes, with only a clear departure from that trend at ${ }^{203} \mathrm{Fr}$. Meanwhile, Voss et al. concluded that this departure was already visible in ${ }^{206} \mathrm{Fr}$. It is suggested that the systematic uncertainty on both data sets does not allow any conclusion on ${ }^{206} \mathrm{Fr}$ to be unambiguously drawn and that other experimental approaches should be sought to address this question.

\section{B. Spins and structure of ${ }^{202} \mathrm{At}$ and ${ }^{198} \mathrm{Bi}$}

Using the data presented in Table II, the partial decay widths were extracted according to the formalism of Rassmussen [43]. The hindrance factors were then calculated in comparison to a pair of neighboring even- $Z$, even- $N$ isotopes. For ${ }^{206} \mathrm{Fr}$, the pair ${ }^{204,206} \mathrm{Rn}$ was chosen, with partial decay widths of $49.4(14) \mathrm{keV}$ and $39.7(24) \mathrm{keV}$, respectively. For ${ }^{202} \mathrm{At}$, the pair ${ }^{200,202}$ Po was chosen, with partial decay widths of 29.7(10) $\mathrm{keV}$ and 25(9) $\mathrm{keV}$, respectively. The hindrance factors are shown in Table II.

From the low hindrance factor in the decay of each state in ${ }^{206} \mathrm{Fr}$ and ${ }^{202} \mathrm{At}$, with values around 2 and the highest only reaching 4 , it can be concluded that all these decays are unhindered and proceed between states with the same spin-parity, and similar nuclear configurations. This means that the three states in ${ }^{202} \mathrm{At}$ and in ${ }^{198} \mathrm{Bi}$ have respective spins $3^{(+)}, 7^{(+)}$, and $10^{(-)}$, as shown in Fig. 2.

This suggests that the orbital occupancy of these states follows that discussed above for ${ }^{206} \mathrm{Fr}$, namely the $\left(\pi 1 h_{9 / 2} \otimes\right.$ $\left.v 3 p_{3 / 2}\right)_{3^{+}}, \quad\left(\pi 1 h_{9 / 2} \otimes v 2 f_{5 / 2}\right)_{7^{+}}$, and $\left(\pi 1 h_{9 / 2} \otimes v 1 i_{13 / 2}\right)_{10^{-}}$ configurations. Note that the slightly higher hindrance factor in the decay of the $3^{(+)}$state in ${ }^{202}$ At could be due to a higher admixture of the $\left(\pi 1 h_{9 / 2} \otimes \nu 2 f_{5 / 2}\right)_{3^{+}}$configuration in ${ }^{198} \mathrm{Bi}$. A direct measurement of the magnetic dipole moment of this isotope with laser spectroscopy could provide more information on that effect. Unpublished data from the IRIS facility, Gachina, Russia, cover the region of interest [44].

\section{SUMMARY AND OUTLOOK}

We have performed high-resolution collinear resonance ionization spectroscopy on a beam of ${ }^{206} \mathrm{Fr}$ with the use of
CRIS at CERN-ISOLDE. The combination of high-resolution laser spectroscopy and decay spectroscopy was essential in the unambiguous assignment of the different hyperfine structure components.

The study of these hyperfine structure components has allowed confirmation of previous findings, namely that the three states are best described as $\left(\pi 1 h_{9 / 2} \otimes \nu 3 p_{3 / 2}\right)_{3^{+}},\left(\pi 1 h_{9 / 2} \otimes\right.$ $\left.v 2 f_{5 / 2}\right)_{7^{+}}$, and $\left(\pi 1 h_{9 / 2} \otimes v 1 i_{13 / 2}\right)_{10^{-}}$configurations.

The isomer shifts between the different data sets of ${ }^{206} \mathrm{Fr}$ are in good agreement; however, the changes in the mean-square charge radii relative to ${ }^{221} \mathrm{Fr}$ differ substantially and may lead to different conclusions. This is due to large systematic uncertainties related to the atomic parameters, and care should be taken in extracting nuclear information in light of these discrepancies.

The study of the $\alpha$-decay spectra has allowed the extraction of branching ratios in unprecedented clean conditions. The decay of the two long-lived states in ${ }^{206} \mathrm{Fr}$ could be disentangled and the branching ratios of the $3^{(+)}$and $10^{(-)}$states in ${ }^{206} \mathrm{Fr}$ determined for the first time. The branching ratios in the $\alpha$ decay of ${ }^{202}$ At could also be extracted.

Based on this new information, the hindrance factors in the ${ }^{206} \mathrm{Fr}-{ }^{202} \mathrm{At}-{ }^{198} \mathrm{Bi}$ decay chain could be calculated. From the low hindrance factor, the spins of the states in ${ }^{202}$ At and ${ }^{198} \mathrm{Bi}$ could be determined, as well as the major configurations of the different states. In the case of the $3^{(+)}$state in ${ }^{198} \mathrm{Bi}$, it is suggested that an increased admixture of the $\left(\pi 1 h_{9 / 2} \otimes\right.$ $\left.v 2 f_{5 / 2}\right)_{3}+$ configuration is responsible for a slightly higher hindrance factor.

\section{ACKNOWLEDGMENTS}

We acknowledge the support of the ISOLDE Collaboration and technical teams, and the GSI target laboratory for producing the carbon foils. We are grateful to the COLLAPS Collaboration for the use of their cw TiSa laser system and Wavetrain doubling unit. This work was supported by the IUAP-Belgian State Science Policy (BRIX network P7/12), FWO-Vlaanderen (Belgium), and GOA's 10/010 and 10/05 from KU Leuven; the Science and Technology Facilities Council Consolidated Grant No. ST/F012071/1, Continuation Grant No. ST/J000159/1, and Ernest Rutherford Grant No. ST/L002868/1; ERC Consolidator Grant No. 648381; and the EU's Seventh Framework through ENSAR No. 506065. K.M.L. was supported by the FWO Pegasus Marie Curie Fellowship under Grant No. 267216. T.E.C. was supported by the STFC Ernest Rutherford Fellowship No. ST/J004189/1. K.T.F. was supported by STFC Advanced Fellowship Scheme Grant No. ST/G006415/1. We acknowledge financial aid from the Ed Schneiderman Fund at New York University.
[1] L. P. Gaffney et al., Nature (London) 497, 199 (2013).

[2] I. Budinčević, J. Billowes, M. L. Bissell, T. E. Cocolios, R. P. de Groote, S. De Schepper, V. N. Fedosseev, K. T. Flanagan, S. Franchoo, R. F. Garcia Ruiz, H. Heylen, K. M. Lynch, B. A. Marsh, G. Neyens, T. J. Procter, R. E. Rossel, S. Rothe, I.
Strashnov, H. H. Stroke, and K. D. A. Wendt, Phys. Rev. C 90, 014317 (2014).

[3] K. Heyde and J. L. Wood, Rev. Mod. Phys. 83, 1467 (2011).

[4] A. N. Andreyev et al., Nature (London) 405, 430 (2000).

[5] H. De Witte, A. N. Andreyev, N. Barré, M. Bender, T. E. Cocolios, S. Dean, D. Fedorov, V. N. Fedoseyev, L. M. Fraille, 
S. Franchoo, V. Hellemans, P. H. Heenen, K. Heyde, G. Huber, M. Huyse, H. Jeppesen, U. Köster, P. Kunz, S. R. Lesher, B. A. Marsh, I. Mukha, B. Roussière, J. Sauvage, M. Seliverstov, I. Stefanescu, E. Tengborn, K. Van de Vel, J. Van de Walle, P. Van Duppen, and Y. Volkov, Phys. Rev. Lett. 98, 112502 (2007).

[6] J. Elseviers, A. N. Andreyev, S. Antalic, A. Barzakh, N. Bree, T. E. Cocolios, V. F. Comas, J. Diriken, D. Fedorov, V. N. Fedosseyev, S. Franchoo, J. A. Heredia, M. Huyse, O. Ivanov, U. Köster, B. A. Marsh, R. D. Page, N. Patronis, M. Seliverstov, I. Tsekhanovich, P. Van den Bergh, J. Van De Walle, P. Van Duppen, M. Venhart, S. Vermote, M. Veselský, and C. Wagemans, Phys. Rev. C 84, 034307 (2011).

[7] N. Bree, K. Wrzosek-Lipska, A. Petts, A. Andreyev, B. Bastin, M. Bender, A. Blazhev, B. Bruyneel, P. A. Butler, J. Butterworth, M. P. Carpenter, J. Cederkäll, E. Clément, T. E. Cocolios, A. Deacon, J. Diriken, A. Ekström, C. Fitzpatrick, L. M. Fraile, C. Fransen, S. J. Freeman, L. P. Gaffney, J. E. García-Ramos, K. Geibel, R. Gernhäuser, T. Grahn, M. Guttormsen, B. Hadinia, K. Hadyńska-Kleķ, M. Hass, P.-H. Heenen, R.-D. Herzberg, H. Hess, K. Heyde, M. Huyse, O. Ivanov, D. G. Jenkins, R. Julin, N. Kesteloot, T. Kröll, R. Krücken, A. C. Larsen, R. Lutter, P. Marley, P. J. Napiorkowski, R. Orlandi, R. D. Page, J. Pakarinen, N. Patronis, P. J. Peura, E. Piselli, P. Rahkila, E. Rapisarda, P. Reiter, A. P. Robinson, M. Scheck, S. Siem, K. Singh Chakkal, J. F. Smith, J. Srebrny, I. Stefanescu, G. M. Tveten, P. Van Duppen, J. Van de Walle, D. Voulot, N. Warr, F. Wenander, A. Wiens, J. L. Wood, and M. Zielińska, Phys. Rev. Lett. 112, 162701 (2014).

[8] G. Ulm, S. K. Bhattacherjee, P. Dabkiewicz, G. Huber, H. J. Kluge, T. Kühl, H. Lochmann, E.-W. Otten, K. Wendt, S. A. Ahmad, W. Klempt, R. Neugart, ISOLDE Collaboration, Zeitschrift für Physik A Atomic Nuclei 325, 247 (1986).

[9] T. E. Cocolios, W. Dexters, M. D. Seliverstov, A. N. Andreyev, S. Antalic, A. E. Barzakh, B. Bastin, J. Büscher, I. G. Darby, D. V. Fedorov, V. N. Fedosseyev, K. T. Flanagan, S. Franchoo, S. Fritzsche, G. Huber, M. Huyse, M. Keupers, U. Köster, Y. Kudryavtsev, E. Mané, B. A. Marsh, P. L. Molkanov, R. D. Page, A. M. Sjoedin, I. Stefan, J. Van de Walle, P. Van Duppen, M. Venhart, S. G. Zemlyanoy, M. Bender, and P.-H. Heenen, Phys. Rev. Lett. 106, 052503 (2011).

[10] M. D. Seliverstov, T. E. Cocolios et al., Phys. Lett. B 719, 362 (2013).

[11] J. Stanja, C. Borgmann, J. Agramunt, A. Algora, D. Beck, K. Blaum, C. Böhm, M. Breitenfeldt, T. E. Cocolios, L. M. Fraile, F. Herfurth, A. Herlert, M. Kowalska, S. Kreim, D. Lunney, V. Manea, E. Minaya Ramirez, S. Naimi, D. Neidherr, M. Rosenbusch, L. Schweikhard, G. Simpson, F. Wienholtz, R. N. Wolf, and K. Zuber, Phys. Rev. C 88, 054304 (2013).

[12] M. Venhart et al., Phys. Lett. B 695, 82 (2011).

[13] K. Wallmeroth et al., Nucl. Phys. A 493, 224 (1989).

[14] C. N. Davids, P. J. Woods, H. T. Penttilä, J. C. Batchelder, C. R. Bingham, D. J. Blumenthal, L. T. Brown, B. C. Busse, L. F. Conticchio, T. Davinson, D. J. Henderson, R. J. Irvine, D. Seweryniak, K. S. Toth, W. B. Walters, and B. E. Zimmerman, Phys. Rev. Lett. 76, 592 (1996).

[15] H. Kettunen et al., Eur. Phys. J. A 16, 457 (2003).

[16] Z. Kalaninová, A. N. Andreyev, S. Antalic, F. P. Heßberger, D. Ackermann, B. Andel, M. C. Drummond, S. Hofmann, M. Huyse, B. Kindler, J. F. W. Lane, V. Liberati, B. Lommel, R. D. Page, E. Rapisarda, K. Sandhu, S. Saro, A. Thornthwaite, and P. Van Duppen, Phys. Rev. C 87, 044335 (2013).
[17] J. Uusitalo, J. Sarén, S. Juutinen, M. Leino, S. Eeckhaudt, T. Grahn, P. T. Greenlees, U. Jakobsson, P. Jones, R. Julin, S. Ketelhut, A.-P. Leppänen, M. Nyman, J. Pakarinen, P. Rahkila, C. Scholey, A. Semchenkov, J. Sorri, A. Steer, and M. Venhart, Phys. Rev. C 87, 064304 (2013).

[18] M. Huyse, P. Decrock, P. Dendooven, G. Reusen, P. Van Duppen, and J. Wauters, Phys. Rev. C 46, 1209 (1992).

[19] K. M. Lynch, J. Billowes, M. L. Bissell, I. Budinčević, T. E. Cocolios, R. P. De Groote, S. De Schepper, V. N. Fedosseev, K. T. Flanagan, S. Franchoo, R. F. Garcia Ruiz, H. Heylen, B. A. Marsh, G. Neyens, T. J. Procter, R. E. Rossel, S. Rothe, I. Strashnov, H. H. Stroke, and K. D. A. Wendt, Phys. Rev. X 4, 011055 (2014).

[20] A. Voss, F. Buchinger, B. Cheal, J. E. Crawford, J. Dilling, M. Kortelainen, A. A. Kwiatkowski, A. Leary, C. D. P. Levy, F. Mooshammer, M. L. Ojeda, M. R. Pearson, T. J. Procter, and W. A. Tamimi, Phys. Rev. C 91, 044307 (2015).

[21] R. Collister, G. Gwinner, M. Tandecki, J. A. Behr, M. R. Pearson, J. Zhang, L. A. Orozco, S. Aubin, and E. Gomez (FrPNC Collaboration), Phys. Rev. A 90, 052502 (2014).

[22] J. Zhang, M. Tandecki, R. Collister, S. Aubin, J. A. Behr, E. Gomez, G. Gwinner, L. A. Orozco, M. R. Pearson, and G. D. Sprouse (FrPNC Collaboration), Phys. Rev. Lett. 115, 042501 (2015).

[23] K. T. Flanagan, K. M. Lynch, J. Billowes, M. L. Bissell, I. Budinčević, T. E. Cocolios, R. P. de Groote, S. De Schepper, V. N. Fedosseev, S. Franchoo, R. F. Garcia Ruiz, H. Heylen, B. A. Marsh, G. Neyens, T. J. Procter, R. E. Rossel, S. Rothe, I. Strashnov, H. H. Stroke, and K. D. A. Wendt, Phys. Rev. Lett. 111, 212501 (2013).

[24] R. P. de Groote, I. Budinčević, J. Billowes, M. L. Bissell, T. E. Cocolios, G. J. Farooq-Smith, V. N. Fedosseev, K. T. Flanagan, S. Franchoo, R. F. Garcia Ruiz, H. Heylen, R. Li, K. M. Lynch, B. A. Marsh, G. Neyens, R. E. Rossel, S. Rothe, H. H. Stroke, K. D. A. Wendt, S. G. Wilkins, and X. Yang, Phys. Rev. Lett. 115, 132501 (2015).

[25] E. Kugler, Hyperfine Interact. 129, 23 (2000).

[26] T. J. Procter et al., J. Phys. Conf. Ser. 381, 012070 (2012).

[27] T. E. Cocolios et al., Nucl. Instrum. Methods Phys. Res., Sect. B 317, 565 (2013).

[28] T. E. Cocolios et al., Nucl. Instrum. Methods Phys. Res., Sect. B (2015).

[29] J. E. Sansonetti, J. Phys. Chem. Ref. Data 36, 497 (2007).

[30] M. M. Rajabali, K. M. Lynch, T. E. Cocolios et al., Nucl. Instrum. Methods Phys. Res., Sect. A 707, 35 (2013).

[31] K. M. Lynch, T. E. Cocolios, N. Althubiti, G. J. Farooq-Smith, and A. J. Smith (unpublished).

[32] A. Voss, M. R. Pearson, J. Billowes, F. Buchinger, B. Cheal, J. E. Crawford, A. A. Kwiatkowski, C. D. P. Levy, and O. Shelbaya, Phys. Rev. Lett. 111, 122501 (2013).

[33] E. Gomez, S. Aubin, L. A. Orozco, G. D. Sprouse, E. IskrenovaTchoukova, and M. S. Safronova, Phys. Rev. Lett. 100, 172502 (2008)

[34] A. Coc et al., Phys. Lett. B 163, 66 (1985).

[35] B. K. Sahoo, D. K. Nandy, B. P. Das, and Y. Sakemi, Phys. Rev. A 91, 042507 (2015).

[36] V. A. Dzuba, W. R. Johnson, and M. S. Safronova, Phys. Rev. A 72, 022503 (2005).

[37] S. Zhu and F. Kondev, Nucl. Data Sheets 109, 699 (2008).

[38] F. G. Kondev, Nucl. Data Sheets 109, 1527 (2008). 
[39] H. De Witte et al., Eur. Phys. J. A 23, 243 (2005).

[40] T. E. Cocolios et al., J. Phys. G 37, 125103 (2010).

[41] J. F. Ziegler, M. D. Ziegler, and J. P. Biersack, Nucl. Instrum. Methods Phys. Res., Sect. B 268, 1818 (2010).
[42] J. Wouters et al., J. Phys. G: Nucl. Part. Phys. 17, 1673 (1991). [43] J. O. Rasmussen, Phys. Rev. 113, 1593 (1959).

[44] P. Campbell, I. D. Moore, and M. R. Pearson, Prog. Part. Nucl. Phys. 86, 127 (2016). 